\title{
Research on Distribution Automation Based on Power Supply Reliability
}

Shuai Du

Nanjing University of Science and Technology Institute of Automation, Nanjing, Jiangsu 210094, China. E-mail: shuaiddus@yeah.net

The development of economy can not be separated from sufficient energy supply. With the progress of technology, the distribution network gradually expands the capacity and transportation, but also brings security risks. The distribution automation system can effectively manage the power grid faults. This paper briefly introduced the calculation model of the economic cost and the reliability rate of the distribution automation, and then the assembly scheme of the distribution terminal was optimized by using the improved binary particle swarm optimization (PSO) algorithm. Next, the simulation analysis of the distribution terminal distribution on a $10 \mathrm{KV}$ main feeder line in Xuanwu District of Nanjing city was carried out in MATLAB software. The results showed that the improved binary PSO algorithm could optimize the assembly scheme of remote metering and remote signalling and the assembly scheme of remote metering, remote signalling and remote control rapidly; the hybrid assembly scheme needed a little more time; the power supply reliability rate of the optimized three terminal assembly schemes was over $99 \%$; the hybrid assembly scheme had higher power supply reliability rate and the lowest economic cost.

Keywords: Distribution Automation, Economic Cost, Power Supply Reliability, Particle Swarm Optimization

\section{Introduction}

The rapid development of Chinese industrial economy is inseparable from the huge and stable energy supply, and electric energy is one of them [1]. The demand of industrial development and daily life makes the power grid system continue to expand. Although the transportation volume of power improves, it also increases the distribution risk [2]. The grid failure will affect daily production and life in a large range due to the high dependence of modern society on electric power, which will not only cause economic losses, but also greatly reduces the happiness of residents. Therefore, the timely maintenance of power grid fault is conducive to the security of power grid [3]. In addition to regular manual maintenance, the means of detecting power grid also include intelligent automatic fault detection means, such as highvoltage circuit breaker. The power grid security technology discussed in this paper is distribution automation technolo-gy, which can quickly locate and isolate the fault point and realize power supply recovery. Onen et al. [4] put forward a series of analysis and on-site verification, evaluated the efficiency, reliability and capacity improvement of the model centered distribution automation, and found that the model centered distribution automation had a positive impact on the system efficiency, capacity and reliability. Jamali et al. [5] attempted to find fault location using the iterative algo-rithm based on state estimation. The simulation results showed that the algorithm could accurately locate the fault in distribution network, which provides a data support for distribution automation. Liu et al. [6] proposed a power supply capacity evaluation method of medium voltage distribution system considering power supply reliability. In this paper, the calculation model of the economic cost and the reliability rate of the distribution automation is briefly introduced, and the assembly scheme of the distribution terminal was optimized by using the improved binary particle swarm op-timization (PSO) algorithm. Then, the simulation analysis of the distribution terminal distribution on a $10 \mathrm{KV}$ main feeder in Xuanwu District of Nanjing city was carried out in MATLAB software [7].

\section{Distribution automation and planning}

\subsection{Distribution automation system}

The distribution automation system is a system that combines the distribution network architecture with the related monitoring equipment terminals and realizes the distribution network control by using the communication network through the overall planning procedure. The distribution automation system has different types according to the diver-sity of functions, but its basic structure is similar. As shown in Fig. 1, the basic structure of different types of distribution automation system is divided into three parts after the diversified functions are removed [8]. The distribution terminal is an important component of the distribution automation system, and its main function is to collect the operation data of the current distribution network and upload the fault to the distribution main station.

The functions of the distribution terminal include remote metering, remote signalling and remote control. Remote me-tering and remote signaling mean transmitting the circuit data and the operation information of the power grid hard-ware facilities obtained by the terminal from the power grid detection to the main station after coding. Remote control means that the main station makes feedback according to the collected information and makes remote control on the terminal. According to the function type [9] of the terminal, it can be divided into two types, two-remote terminal and three-remote terminal. 


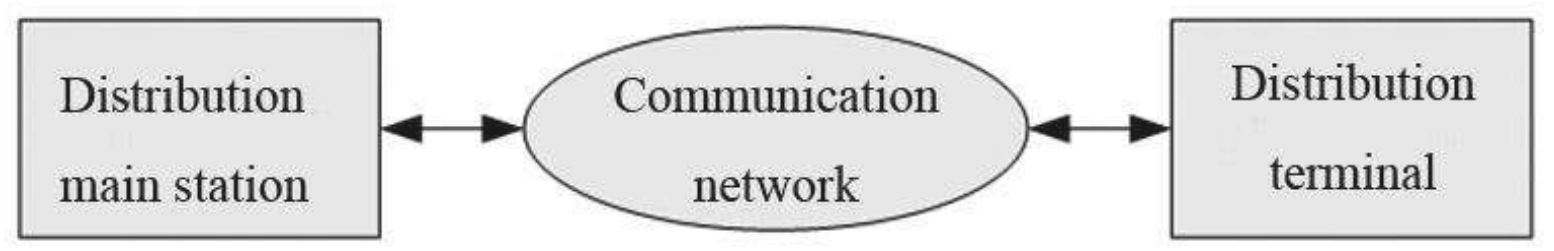

Fig. 1 Diagram of distribution automation

\subsection{Distribution automation planning}

The indicator of evaluating whether the terminal configuration of the distribution automation system is reasonable or not is the whole life cycle cost and power supply reliability. The whole life cycle cost is the construction and operation cost of the distribution automation system, and its mathematical model [10] is:

$$
\left\{\begin{array}{l}
C_{\text {all }}=C_{F}+C_{M}+C_{D}-C_{I} \\
C_{F}=N_{k} C_{f} \frac{q(1+q)^{p}}{(1+q)^{p}-1} \\
C_{M}=N_{k} C_{p} C_{f} \\
C_{D}=N_{k} C_{t} C_{f} \\
C_{I}=C_{s} W_{E N S} \\
W_{E N S}=8760 \sum P_{i} \eta
\end{array}\right.
$$

Where:

$C_{\text {all }}$...Total input cost,

$C_{F}$...Terminal investment cost,

$C_{M}$...Operating cost,

$C_{D}$...Cost of terminal disposal,

$C_{I}$...Loss of users,

$N_{k}$..Total number of terminals in the grid,

$C_{f}$...Cost of a single terminal,

$q$...Discount rate,

$p$...Service life of the terminal,

$C_{p}$...Operation conversion rate,

$C_{t}$...Conversion rate of waste treatment cost,

$C_{s}$...Income of unit electric energy,

$W_{E N S} \ldots$...ncome brought by the increase of power generation after distribution automation,

$P_{i} \ldots$ Load in section $\mathrm{i}$ of the grid,

$\eta_{i} \ldots$ Load rate in section $\mathrm{i}$.

The reliability of power supply is evaluated by the outage time. In case of power failure in a certain area of the power grid, the duration of power failure is:

$$
T=t_{1}+t_{2}+t_{3}
$$

Where:

$T$...Duration of the outage,

$t_{1}$... Troubleshooting time of the fault point,

$t_{2}$...Isolation time of the fault point,
$t_{3}$...Repair time of the failure point.

After adopting the distribution automation system, the power supply reliability of the power grid can be improved. The calculation model of power supply reliability [11] is as follows.

(1) Two-remote terminals are installed: two-remote terminals have remote metering and remote signaling functions, so it is not necessary to spend time on troubleshooting the fault points, but it is still necessary to manually isolate and repair the fault points. Then the calculation formula is:

$$
\alpha=1-\frac{(k+1) F t_{2}+\sum_{i=1}^{k+1} t_{3} f_{i}}{8760(k+1)},
$$

Where:

$\alpha$...Power supply reliability,

$k$...Number of terminal installed,

$F t_{2}$...Outage hours $\left(t_{2}\right)$ before the fault points are isolated,

$F$...Equivalent failure rate,

$f_{i}$...Failure rate of section $\mathrm{i}$.

(2) Three-remote terminals are installed: three-remote terminals also have additional remote control function. After detecting the fault, in addition to rapidly feeding back to the main station, they will also quickly isolate the fault point, so it does not need to spend time to check and isolate the fault point, but just need to repair the fault point manually. Then the calculation formula is:

$$
\alpha=1-\frac{\sum_{i=1}^{k+1} t_{3} f_{i}}{8760(k+1)} \text {. }
$$

(3) Mixed installation of two-remote and three-remote terminals: after the mixed installation of two-remote and three-remote terminals, manual repair is needed no matter whether the fault point is isolated after being detected by the two kinds of terminals; therefore the two terminals can be regarded as one in the processing time of repairing the fault, while the isolation time of the fault point needs to consider the common influence of two-remote and three-remote terminals. Therefore, the calculation formula is:

$$
\alpha=1-\frac{F t_{2}}{8760\left(k_{1}+1\right)}-\frac{\sum_{i=1}^{k+1} t_{3} f_{i}}{8760(k+1)},
$$

Where:

$k_{1}$...Number of three-remote terminals. 


\section{Improved PSO algorithm}

As mentioned above, calculating the configuration scheme of terminals is a problem of objective optimization. If the scheme is enumerated manually, it will take time and effort. In this study, PSO algorithm was used to optimize the configuration scheme.

PSO algorithm [12] is an evolutionary algorithm inspired by the phenomenon of birds foraging in nature. A $\mathrm{N}$-dimensional search space is set, and the scale of the particle swarm is $\mathrm{M}$, then its iterative algorithm formula is:

$$
\left\{\begin{array}{l}
v_{i, j}(t+1)=v_{i, j}(t)+c_{1} r_{1 i, j}(t)\left(P_{i, j}(t)-X_{i, j}(t)\right)+c_{2} r_{2 i, j}(t)\left(G_{i, j}(t)-X_{i, j}(t)\right) \\
X_{i, j}(t+1)=v_{i, j}(t+1)+X_{i, j}(t) \\
X_{i}(t)=\left[X_{i, 1}(t), X_{i, 2}(t), \cdots X_{i, j}(t), \cdots X_{i, N}(t)\right] \\
v_{i}(t)=\left[v_{i, 1}(t), v_{i, 2}(t), \cdots v_{i, j}(t), \cdots v_{i, N}(t)\right] \\
P_{i}(t)=\left[P_{i, 1}(t), P_{i, 2}(t), \cdots P_{i, j}(t), \cdots P_{i, N}(t)\right] \\
i \in[1, M], j \in[1, N]
\end{array},\right.
$$

Where:

$X_{i}(t)$...Position of the i-th particle before adjustment,

$v_{i}(t)$...Velocity of the i-th particle before adjustment,

$P_{i}(t)$...Individual optimal position of the $\mathrm{i}$-th particle before adjustment,

$X_{i, j}(t)$...Position of the $\mathrm{i}$-th particle before the $\mathrm{j}$-dimensional adjustment

$X_{i, j}(t+1) \ldots$ Position of the $\mathrm{i}$-th particle after the $\mathrm{j}$-dimensional adjustment,

$v_{i, j}(t) \ldots$ Velocity of the $\mathrm{i}$-th particle before the $\mathrm{j}$-dimensional adjustment,

$v_{i, j}(t+1) \ldots$ Velocity of the $\mathrm{i}$-th particle after the $\mathrm{j}$-dimensional adjustment,

$c_{1}$...Learning factor, involving the adjustment of individual optimal position,

$c_{2}$...Learning factor, involving the adjustment of global optimal position,

$r_{1 i, j}$...A random number, evenly distributed in $(0,1)$,

$r_{2 i, j} \ldots$ A random numbers, evenly distributed in $(0,1)$.

In this study, PSO algorithm was used for optimizing the configuration of distribution terminals. In the actual configu-ration, terminals are generally installed on the sectional switches of power grid feeders. Therefore, a series of binary sequences can be used to represent the terminal configuration scheme on a feeder: "1" represents the installation of terminal and " 0 " represents the no installation of terminal. Then the above iterative formula of PSO algorithm needs to make some changes, the iteration formula of its speed is unchanged, the $\mathrm{j}$-dimensional position of particle is limited to 1 or 0 , and the iterative formula of the position is:

$$
\left\{\begin{array}{l}
X_{i, j}(t+1)= \begin{cases}1 & r<S\left(v_{i, j}(t)\right) \\
0 & r \geq S\left(v_{i, j}(t)\right)\end{cases} \\
S\left(v_{i, j}(t)\right)=\frac{1}{1+\exp \left(-v_{i, j}(t)\right)}
\end{array}\right.
$$

Where:

$r$...A uniform random number between 0 and 1 .

Although the above-mentioned PSO algorithm is changed to binary in position, which reduces the calculation amount to a certain extent, it is still a traditional PSO algorithm which is easy to fall into local optimal solution or has the prob-lem of slow convergence in the calculation process. Therefore, the standard binary PSO algorithm is improved:

$$
\left\{\begin{array}{l}
v_{i, j}(t+1)=\omega v_{i, j}(t)+c_{1} r_{1 i, j}(t)\left(P_{i, j}(t)-X_{i, j}(t)\right)+c_{2} r_{2 i, j}(t)\left(G_{i, j}(t)-X_{i, j}(t)\right) \\
\omega=\omega_{\max }-\left(\omega_{\max }-\omega_{\min }\right)\left(\frac{1}{2}\left(1+\sin \left(\pi\left(\frac{t}{T}-\frac{1}{2}\right)\right)\right)\right) \\
X_{i, j}(t+1)= \begin{cases}1 & r<S\left(v_{i, j}(t)\right) \\
0 & r \geq S\left(v_{i, j}(t)\right)\end{cases} \\
S\left(v_{i, j}(t)\right)=\frac{1}{1+\exp \left(-v_{i, j}(t)\right)}
\end{array} .\right.
$$

The improvement of the standard binary PSO algorithm is that inertia weight is added to the velocity itera- tion for-mula, and the inertia weight is nonlinear decreasing [13]. and are the maximum value and minimum value of respectively. 


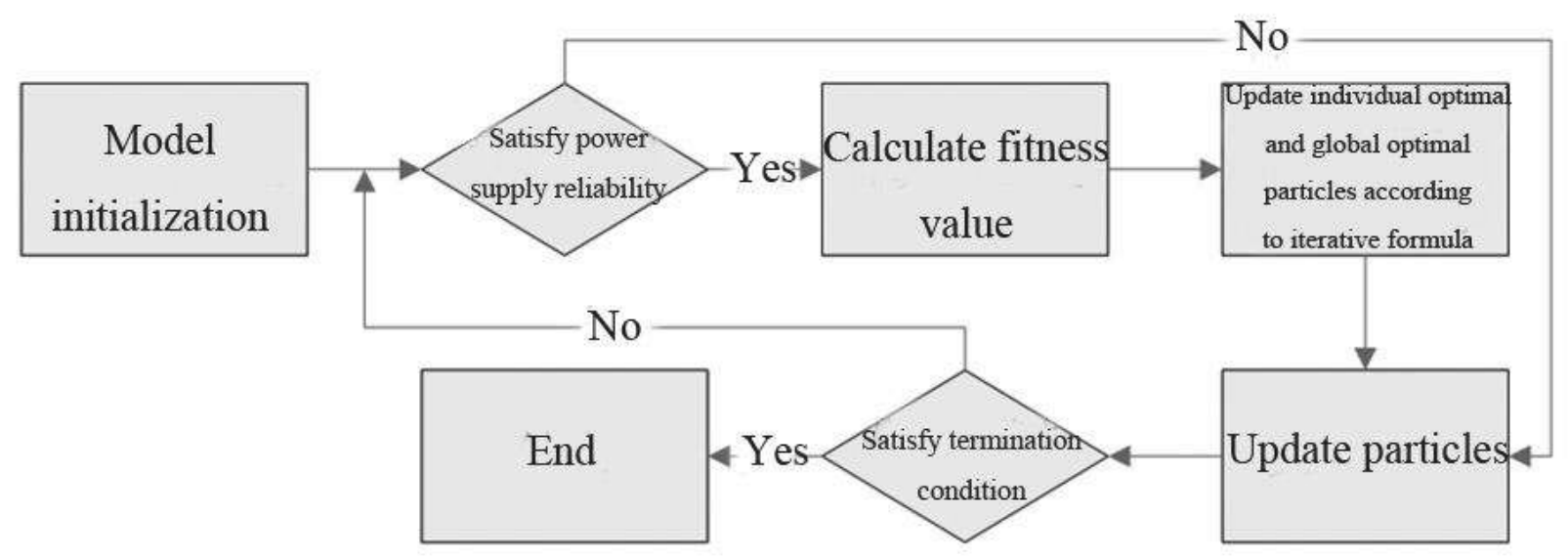

Fig. 2 Optimization process of terminal configuration scheme based on binary PSO algorithm

The optimization process of the terminal configuration scheme of the improved binary PSO algorithm is basically the same, as shown in Fig. 2.

(1) Firstly, the calculation model is initialized, including particle population size, maximum number of iterations [14], etc.

(2) Secondly, initial particles are randomly generated, which are composed of a series of binary sequences, and each particle represents a scheme.

(3) The power supply reliability of each particle is calculated according to the above power supply reliability calculation formula, and the particles which do not meet the power supply reliability requirements are updated to make them meet the power supply reliability requirements.

(4) When the particle swarm meets the requirements of power supply reliability, its fitness value is calculated. The fit-ness value in this study adopts the whole life cycle cost, and its calculation formula is as described above.

(5) Then the individual optimal and global optimal particles are updated according to the fitness value and iterative formula, and all particles in the particle swarm are updated.

(6) After updating, whether the algorithm meets the termination conditions is determined. If it does not meet the condi-tions, it continues to iterate; if it does, the algorithm ends, and the results are output.

\section{Example analysis}

\subsection{Experimental environment}

In this study, the binary PSO algorithm was written by MATLAB software [15], which was used for optimizing the con-figuration scheme of the distribution terminal. The experiment was carried out with a laboratory server. The server configuration included windows7 system, i7 processor and $16 \mathrm{~g}$ memory.

\subsection{Experimental data}

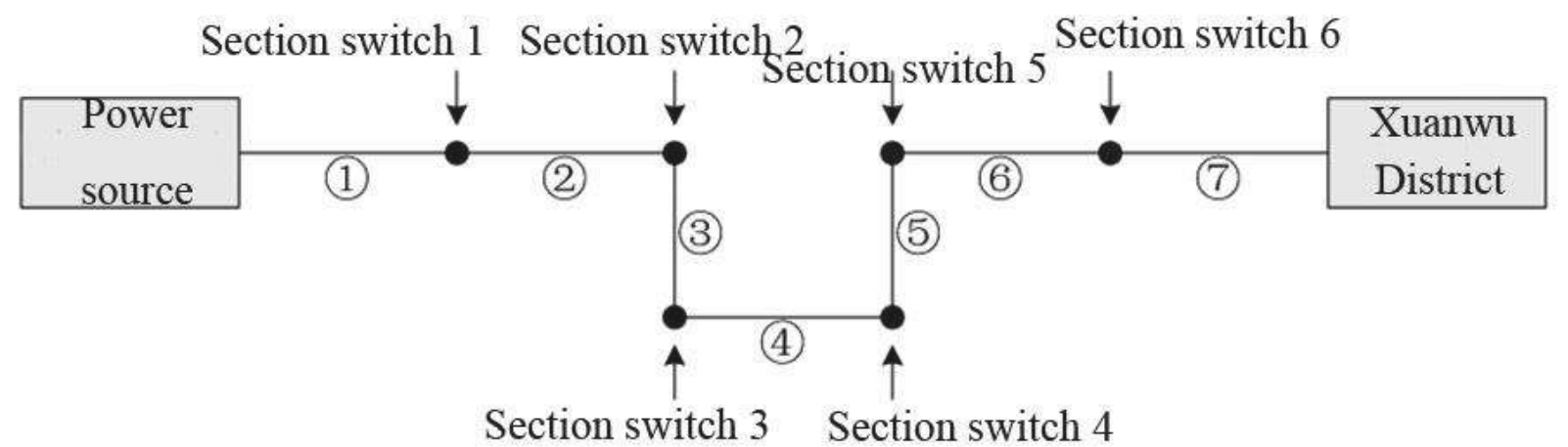

Fig. 3 A simplified drawing of a $10 \mathrm{kV}$ main feeder in Xuanwu District, Nanjing

In this study, a $10 \mathrm{kV}$ main feeder in Xuanwu District of Nanjing city was selected as the simulation analysis target. The original power grid distribution map is relatively complex as it passed through the urban area. In order to facilitate the application of PSO algorithm, it was simplified. As shown in Fig. 3, six section switches in the simplified main feeder can be used to install distribution terminals, and the section switches divide the main feeder into seven areas, the length, number of users and line load of each section are shown in Table 1. In addition, the line type in each area is overhead insulated line [16], and the failure rate of transmission line is 0.11 times/(km*year).

In the period of power failure due to failure of distribution network, the time of troubleshooting was expected 
to be $1 \mathrm{~h}$, the time of fault isolation was expected to be 2 $\mathrm{h}$, and the time of fault maintenance was expected to be 4 $\mathrm{h}$; the cost of installing a single two-remote terminal was 30000 yuan, and the cost of installing a single threeremote terminal was 90000 yuan.

Tab. 1 Relevant parameters of line regions

\begin{tabular}{|l|l|l|l|}
\hline $\begin{array}{l}\text { Line re- } \\
\text { gions }\end{array}$ & Length/km & $\begin{array}{l}\text { Line us- } \\
\text { ers/n }\end{array}$ & $\begin{array}{l}\text { Line } \\
\text { load/kW }\end{array}$ \\
\hline 1 & 1.21 & 6 & 965 \\
\hline 2 & 1.02 & 5 & 251 \\
\hline 3 & 1.16 & 2 & 365 \\
\hline 4 & 2.15 & 4 & 124 \\
\hline 5 & 1.81 & 6 & 987 \\
\hline 6 & 1.21 & 3 & 415 \\
\hline 7 & 0.69 & 4 & 120 \\
\hline
\end{tabular}

Relevant parameters of the improved binary PSO algorithm were as follows. The particle swarm size was 50 , the max-imum times of iterations was 100, and learning factor and were 1.5. The particle coding in particle swarm is different according to the configuration scheme. In the configuration schemes of all two-remote terminals and three-remote terminals, both of them were coded in the same way, with 0 for no installation and 1 for installation, and the length of code was 6 , for example, represents installing terminals at the second and sixty section switches. In the configuration of mixed installation of two-remote and three-remote terminals, a two-digit binary code was used to present whether a section switch was installed and the installation type, 00 and 11 for no installation, 01 for installation of two-remote terminals, and 10 for installation of three-remote terminals, and the length of code was 12 , for example, represents the second and forth section switches installed two-remote terminals.

\subsection{Experimental results}

If the exhaustive method was used to design the terminal configuration scheme, the total number of schemes using two- or three-remote terminals alone was , and moreover there were kinds of schemes of mixed installation of two-remote and three-remote terminals. It is almost unrealistic to calculate the reliability and economic cost of power supply for each scheme. After using the improved binary PSO algorithm, as shown in Fig. 4, only about 20 times of iterations were needed for the assembly scheme of two-remote terminals and the scheme of three-remote terminals to obtain the appropriate terminal assembly scheme. The mixed assembly scheme of two- and threeremote terminals needed 35 times, which greatly reduced the calculation amount. It can also be seen from Fig. 4 that the fitness value of the final convergence of the assembly scheme of two-remote terminals was larger, and the fitness values of the final convergence of the assembly scheme of three-remote terminals and the assembly scheme of two-remote terminals and three-remote terminals were low and close, but the fitness value of the hybrid assembly scheme was smaller.

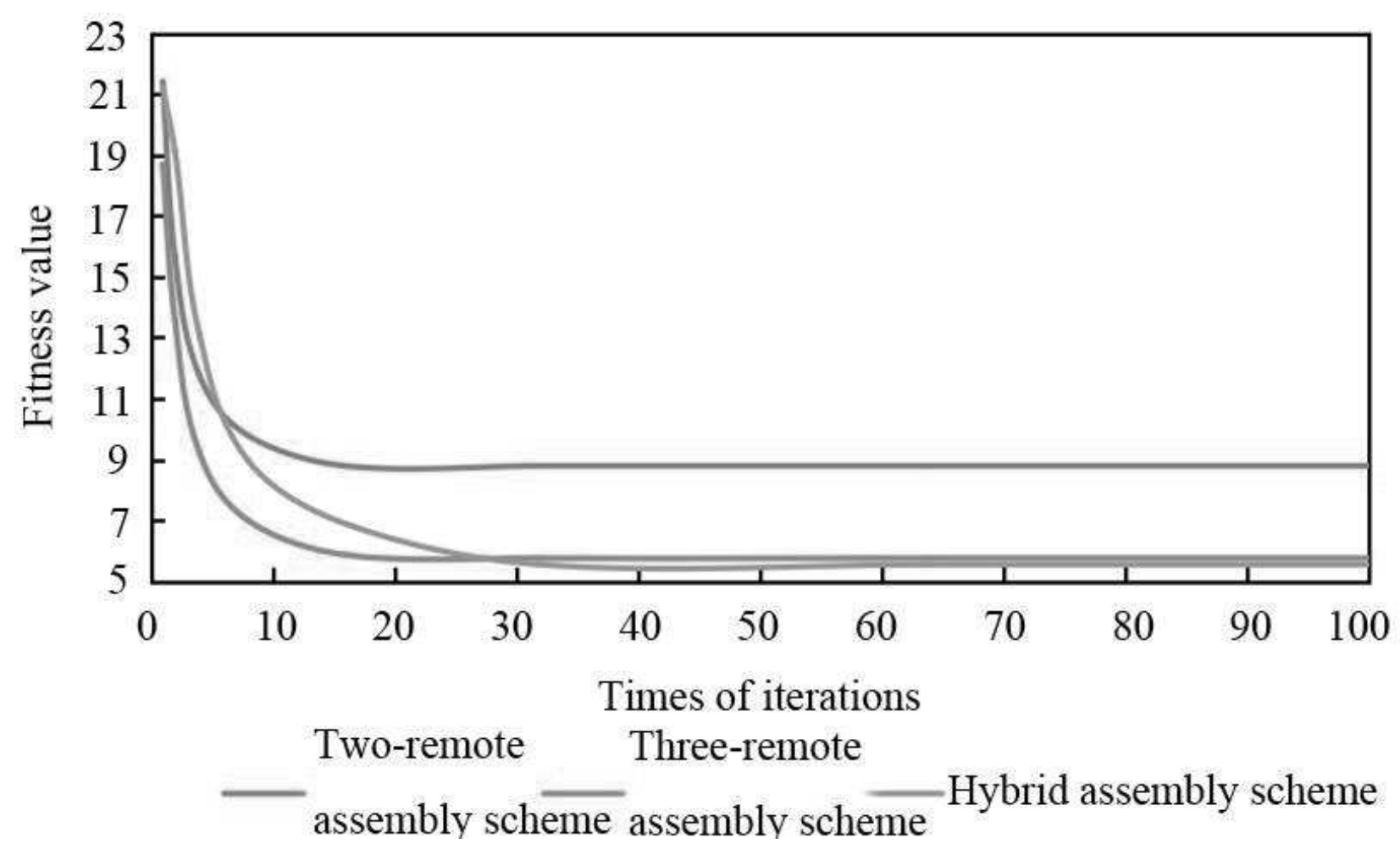

Fig. 4 Convergence of three configuration schemes 


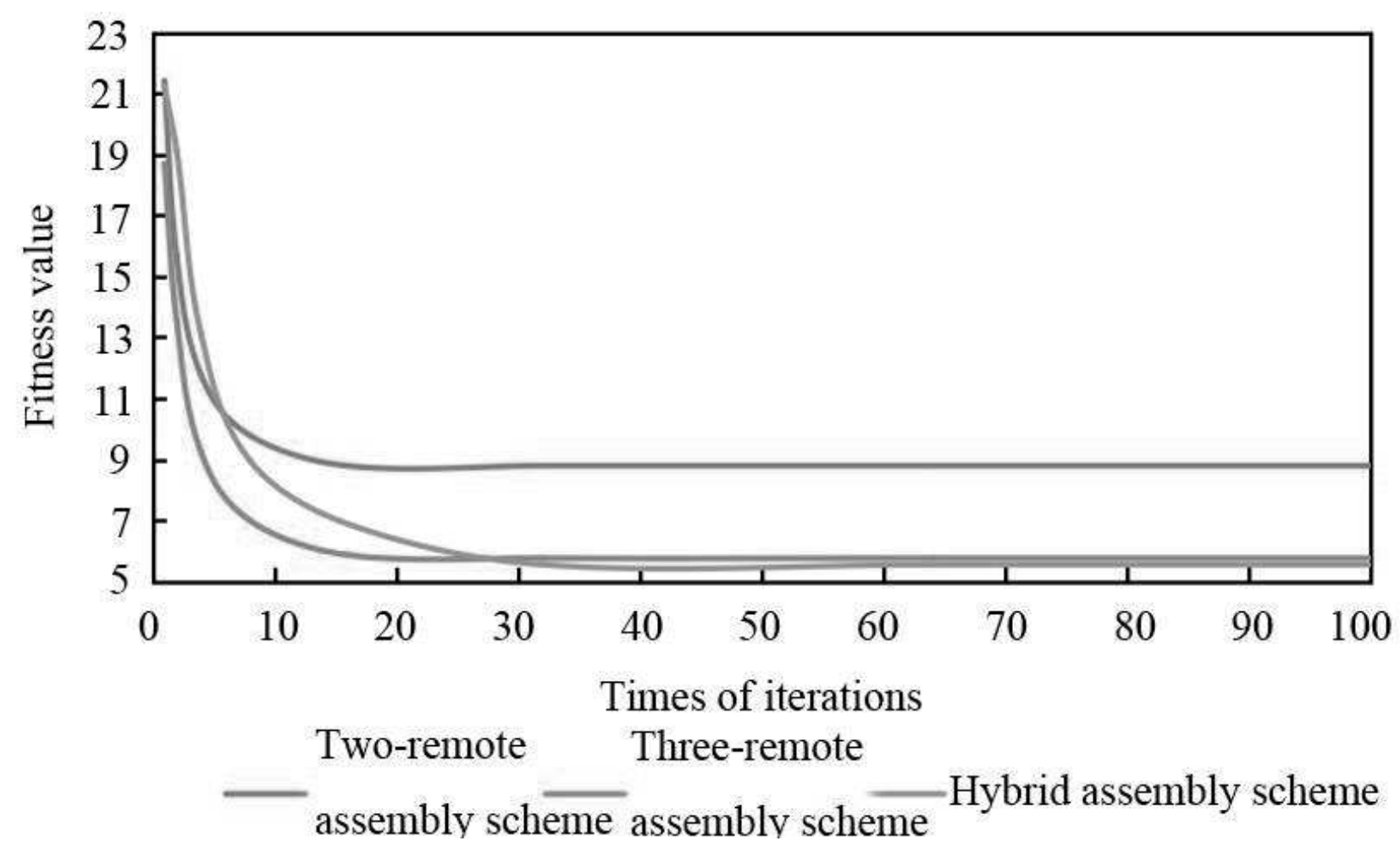

Fig. 5 The economic cost and power supply reliability of the three schemes

After 100 times of iterations, the three assembly schemes all obtained an appropriate terminal allocation scheme. The economic cost and power supply reliability are shown in Fig. 5. The final distribution scheme of the two-remote termi-nal assembly scheme was . In details, three two-remote terminals were installed at the first, fourth and sixth section switches respectively. The economic cost of the scheme was 87800 yuan, and the power supply reliability rate that can be achieved was $99.88 \%$. The final distribution scheme of the three-remote terminal assembly scheme was. Three three-remote terminals were installed and loaded at the second, fourth and sixth section switches re-spectively. The economic cost of the scheme was 57500 yuan, and the power supply reliability rate that can be achieved was $99.97 \%$. The final distribution scheme of the hybrid assembly scheme was . One two-remote terminal and two three-remote terminals were installed. The two-remote terminal was installed at the first sec-tion switch, and the three-remote terminals were installed at the fourth and sixth section switches respectively. The economic cost of the scheme was 55400 yuan, and the power supply reliability rate that can be achieved was $99.98 \%$. It was seen from Fig. 5 and the distribution scheme that the number of terminals required for the three assembly schemes were all three, but the different types and distribution positions made their power supply reliability and eco-nomic cost different. The economic cost of the two-remote terminal assembly schemes was the largest, and the three-remote terminals and hybrid schemes were relatively close and lower than the two-remote terminal assembly scheme. Although the single cost of the two-remote terminal was far lower than that of the threeremote terminal, the high relia-bility of power supply and the shorter outage time brought by the three-remote terminals made the rest of the operation cost greatly reduced, so does the hybrid assembly scheme. The mixed assembly of two-remote and three-remote termi-nals not only kept the high reliability of power supply, but also reduced the terminal cost.

By comparison, the power supply reliability of the three assembly schemes was more than $99 \%$, so it tended to choose the three-remote assembly scheme and hybrid assembly scheme with low cost. The cost and power supply reliability of the hybrid assembly scheme were slightly better than that of the three -remote assembly scheme. Therefore the hybrid assembly scheme was finally selected.

\section{Conclusion}

This paper briefly introduces the calculation model of the economic cost and the reliability rate of the distribution au-tomation and optimizes the assembly scheme of the distribution terminal by using the improved binary PSO algorithm. Then, the simulation analysis of the distribution terminal distribution on a $10 \mathrm{kV}$ main feeder line in Xuanwu District of Nanjing city was carried out in MATLAB software. The results are as follows. Compared with the exhaustive method, the three assembly schemes which applied PSO algorithm obtained the optimal scheme more quickly; the two-remote assembly scheme and three-remote assembly scheme only needed about 20 times of iterations, and the hybrid assem-bly scheme needed about 35 times of iterations. Moreover, the convergence fitness value of the three-remote terminal and hybrid schemes were lower. After 100 times of 
iterations, the optimal two-remote assembly scheme obtained was, the economic cost was 87800 yuan, and the power supply reliability was $99.88 \%$. The optimal threeremote terminal assembly scheme was, the economic cost was 57500 yuan, and the power supply reliability rate was $99.97 \%$. The optimal hybrid assembly scheme was , the economic cost was 55400 yuan, and the relia-bility rate of power supply was $99.98 \%$.

\section{References}

[1] GAN, G., WANG, Z., ZHOU, Y., LI, L., LI, X.Q. (2015). Reliability and Economy Based Closedloop Operation Modes for Medium Voltage Distribution Networks. In: Automation of Electric Power Systems, Vol. 39, No. 16, pp. 144-150.

[2] LI, B., ZHU, J., LI, P.J., WEI, H. (2015). Island Partition of Distribution Network with Unreliable Distributed Generators. In: Automation of Electric Power Systems, Vol. 39, No. 8, pp. 59-65.

[3] SOBOTKA, J., SOLFRONK, P., KOLNEROVA, M., KORECEK, D. (2019). Input Data Acquisition Possibilities for Numerical Simulation of Drawing Process by means of the Contact-less Optical System and Thermo-camera. In: Manufacturing Technology, Vol. 1, pp. 144-149.

[4] ONEN, A., JUNG, J., DILEK, M., CHENG, D., BROADWATER, R.P., SCIRBONA, C., COCKS, G., HAMILTON, S., WANG, X.Y. (2016). Model-centric Distribution Automation: Capacity, Reliability, and Efficiency. In: Electric Power Components and Systems, Vol. 44, No. 5, pp. 1-11.

[5] JAMALI, S., BAHMANYAR, A. (2016). A new fault location method for distribution networks using sparse measurements. In: International Journal of Electrical Power \& Energy Systems, Vol. 81, pp. 459-468.

[6] LIU, H., LI, J., ZHANG, J., SUN, H., LIU, W., QU, G.Q. (2017). Power Supply Capability Evaluation of Medium Voltage Distribution System Considering Reliability. In: Automation of Electric Power Systems, Vol. 41, No. 12, pp. 154-160.

[7] GUO, J., YAO, J., LIU, M., HU, J., SONG, T. (2015). A Routing Algorithm to Prolong Network Lifetime for City Distribution Network. In: Automation of Electric Power Systems, Vol. 39, No. 12, pp. 113-120.
[8] HEIDARI, A., AGELIDIS, V.G., KIA, M., POU, J., AGHAEI, J., SHAFIE-KHAH, M., CATALAO, J.P.S. (2017). Reliability Optimization of Automated Distribution Networks With Probability Customer Interruption Cost Model in the Presence of DG Units. In: IEEE Transactions on Smart Grid, Vol. 8, No. 1, pp. 305-315.

[9] CHEN, D., WU, Z. (2017). Dynamic planning of distribution automation terminal units placement optimization. In: Power System Protection \& Control, Vol. 45, No. 12, pp. 1-8.

[10] SUN, X., ZHOU, Q.J., WANG, B., FU, L.M. (2015). Analysis and Suggestions for Power Supply Reliability Assessment Index of Distribution Network. In: Applied Mechanics and Materials, Vol. 733, pp. 726-729.

[11] ZHANG, E.Z., CHEN, Q.W. (2015). Multi-objective particle swarm optimization for uncertain reliability optimization problems. In: Control \& Decision, Vol. 4, No. 2, pp. 3915 - 3920.

[12] FENG, Z., SI, J., WANG, J. (2016). Research on optimal schedule strategy for active distribution network using particle swarm optimization combined with bacterial foraging algorithm. In: International Journal of Electrical Power \& Energy Systems, Vol. 78, pp. 637-646.

[13] WU, Y.M., XU, Y.X., LUO, L.F., ZHANG, H., ZHAO, X.D. (2018). Research on evolution balancing for product family assembly line in big data environment. In: Manufacturing Technology, Vol. 2, pp. 337-342.

[14] TIAN, D. (2017). Particle Swarm Optimization with Chaos-based Initialization for Numerical Optimization. In: Intelligent Automation \& Soft Computing, Vol. 24, No. 1, pp. 1-12.

[15] QIN, Q., CHENG, S., ZHANG, Q., WEI, Y., SHI, Y. (2015). Multiple strategies based orthogonal design particle swarm optimizer for numerical optimization. In: Computers \& Operations Research, Vol. 60, No. C, pp. 91-110.

[16] SUJOVÁ, E., STŘIHAVKOVÁ, E., ČIERNA, H. (2018). An analysis of the assembly line modernization by using simulation software. In: Manufacturing Technology, Vol. 5, pp. 839-845. 